\title{
THE CO 2-1/1-0 RATIO IN THE DISK AND CENTER OF THE MILKY WAY GALAXY
}

\author{
TETSUO HASEGAWA, JUN-ICHI MORINO, TSUYOSHI SAWADA, \\ TOSHIHIRO HANDA AND KUMIKO SATO \\ Institute of Astronomy, The University of Tokyo \\ Osawa, Mitaka, Tokyo 181, Japan \\ TOMOHARU OKA \\ The Institute of Physical and Chemical Research (RIKEN) \\ Hirosawa, Wako, Saitama 351-01, Japan \\ SEIICHI SAKAMOTO AND KAZUO SORAI \\ Nobeyama Radio Observatory, National Astronomical Observatory \\ Minamimaki, Minamisaku, Nagano 384-13, Japan \\ MASUMICHI SETA \\ Communications Research Laboratories \\ Nukui-kita machi, Koganei, Tokyo 184, Japan \\ MASAHIKO HAYASHI \\ National Astronomical Observatory \\ Osawa, Mitaka, Tokyo 181, Japan \\ LEONARDO BRONFMAN AND JORGE MAY \\ Departamento de Astronomía, Universidad de Chile \\ Casilla 36-D, Santiago, Chile \\ ROY BOOTH AND LARS-ÅKE NYMAN \\ Onsala Space Observatory \\ S-439 92 Onsala, Sweden \\ AND \\ PETER SHAVER \\ European Southern Observatory \\ D-85748 Garching, Germany
}

We are conducting a survey of the CO $J=2-1$ line emission in the southern Milky Way (the Tokyo-Onsala-ESO-Calán Galactic CO survey), using the $60-\mathrm{cm}$ radio telescope (VST2) in La Silla, Chile. It is combined with 


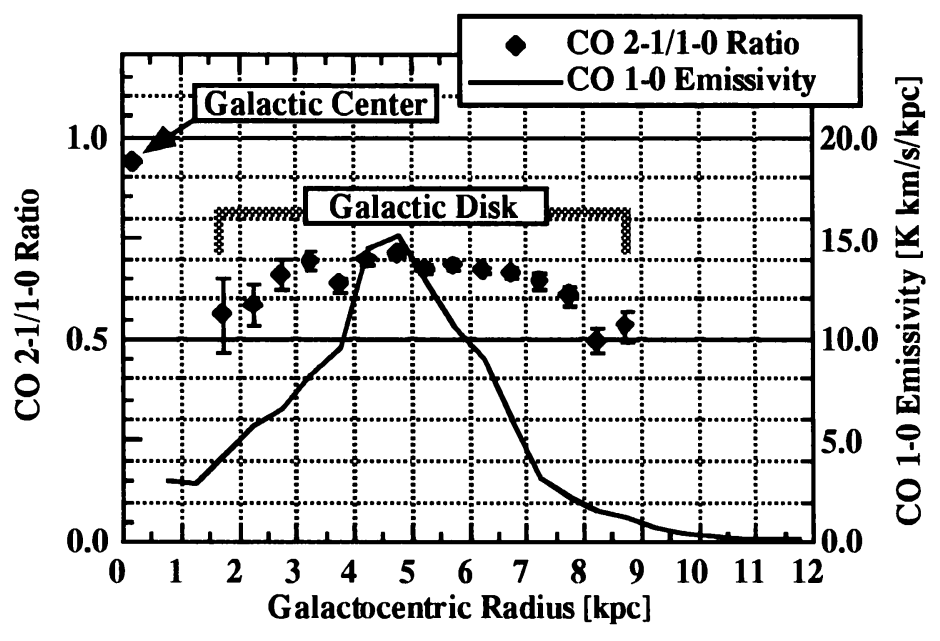

Figure 1. The CO $J=2-1 / J=1-0$ intensity ratio as a function of the Galactocentric radius. The Galactic center value is an average over all emission within $|l| \leq 2.5^{\circ}$ and $|b| \leq 1^{\circ}$. The disk value is from the data at $|l|=5^{\circ} \rightarrow 85^{\circ}$ sampled and analyzed symmetrically for the 1 st and 4 th quadrants. A flat rotation at $\Theta_{0}=220 \mathrm{~km} \mathrm{~s}^{-1}$ was assumed.

the northern survey made with the identical telescope (VST1) at Nobeyama (e.g., Sakamoto et al. 1995), and is directly comparable with the Columbia survey of the CO $J=1-0$ emission (e.g., Dame et al. 1987).

Figure 1 shows the $\mathrm{CO} J=2-1 / J=1-0$ ratio plotted against the Galactocentric radii. The data are from $\mathrm{i}$ ) the galactic plane pilot survey that covers $|l| \leq 90^{\circ}$ sampled at every degree (Hasegawa et al., in preparation), and ii) the new survey of the Galactic center region covering $|l| \leq 6^{\circ}$ and $|b| \leq 2^{\circ}$ (Sawada et al., in preparation). The molecular gas in the central $750 \mathrm{pc}$ of the Galaxy exhibit markedly higher $J=2-1 / J=1-0$ ratio of $0.93 \pm 0.02$, in contrast to the disk gas with the ratio of 0.5-0.7 (total intensity ratio being $0.66 \pm 0.01)$. The Galactic center value in our previous paper (Oka et al. 1997) was underestimated.

\section{References}

Dame, T. M., Ungrechts, H., Cohen, R. S., de Geus, E. J., Grenier, I.A., May, J., Murphy, D. C., Nyman, L. $\AA ., \&$ Thaddeus, P. (1987) A composite CO survey of the entire Milky Way, $A p J$, 322, pp. 706-720

Oka, T., Hasegawa, T., Hayashi, M., Handa, T., \& Sakamoto, S. (1997) CO (J=2-1) line observations of the Galactic center molecular cloud complex. II. Dynamical structure and physical conditions, $A p J$ in press.

Sakamoto, S., Hasegawa, T., Hayashi, M., Handa, T., \& Oka, T. (1995) An out-of-plane CO $(J=2-1)$ survey of the Milky Way. I. The data, $A p J S, 100$, pp. 125-131 